Revue des patrimoines

\title{
Architectures de la petite industrie urbaine : l'exemple des Lilas (Seine-Saint-Denis)
}

Nicolas Pierrot

\section{OpenEdition}

Journals

Édition électronique

URL : http://journals.openedition.org/insitu/3254

DOI : 10.4000/insitu.3254

ISSN : 1630-7305

Éditeur

Ministère de la culture

Référence électronique

Nicolas Pierrot, «Architectures de la petite industrie urbaine : l'exemple des Lilas (Seine-Saint-Denis)», In Situ [En ligne], 8 | 2007, mis en ligne le 01 mars 2007, consulté le 01 mai 2019. URL : http:// journals.openedition.org/insitu/3254; DOI : 10.4000/insitu.3254

Ce document a été généré automatiquement le 1 mai 2019.

\section{(c) $($ i) $(9)$}

In Situ Revues des patrimoines est mis à disposition selon les termes de la licence Creative Commons Attribution - Pas d'Utilisation Commerciale - Pas de Modification 4.0 International. 


\title{
Architectures de la petite industrie urbaine : l'exemple des Lilas (Seine- Saint-Denis)
}

\author{
Nicolas Pierrot
}

\section{Industrialisation, désindustrialisation ${ }^{1}$}

1 La cheminée de l'usine Kalker, "manufacture générale de caoutchouc, amiante et ébonite $»^{2}$, marquait depuis la fin du XIXe siècle, aux portes de Paris, le paysage de la commune (fig. $\mathbf{n}^{\circ}$ 1). Sa destruction en 1968 - une série de photographies témoigne de l'événement ${ }^{3}$ - symbolise l'effacement précoce de l'industrie sur ce territoire ${ }^{4}$. Détruite la même année, l'usine Hemmen et Jacquemin, fondée en 1881, a fait place à la clinique des Lilas 5 . De cette fabrique d'objets en celluloïd (poignées de parapluies) ne subsiste plus aujourd'hui que le logement patronal ${ }^{6}$. Cinq années plus tôt, un ensemble de logements collectifs ${ }^{7}$ succédait aux établissements Patrelle, «fabrique de colorants pour potage obtenus en brûlant des oignons $»^{8}$. Installée aux Lilas avant $1870^{9}$, l'usine occupait dès 1926 plus de $4000 \mathrm{~m}^{2}$ de part et d'autre de la rue du Garde-Chasse ${ }^{10}$. Plus tardivement, en 1982, une partie des ateliers de la Compagnie du Ronéo (meubles de bureau), établis au début des années 1920 au 74-84, boulevard du Général-Leclerc-de-Hauteclocque, en bordure de Romainville, faisait place à 120 logements de « La Demeure familiale »" 
Figure 1

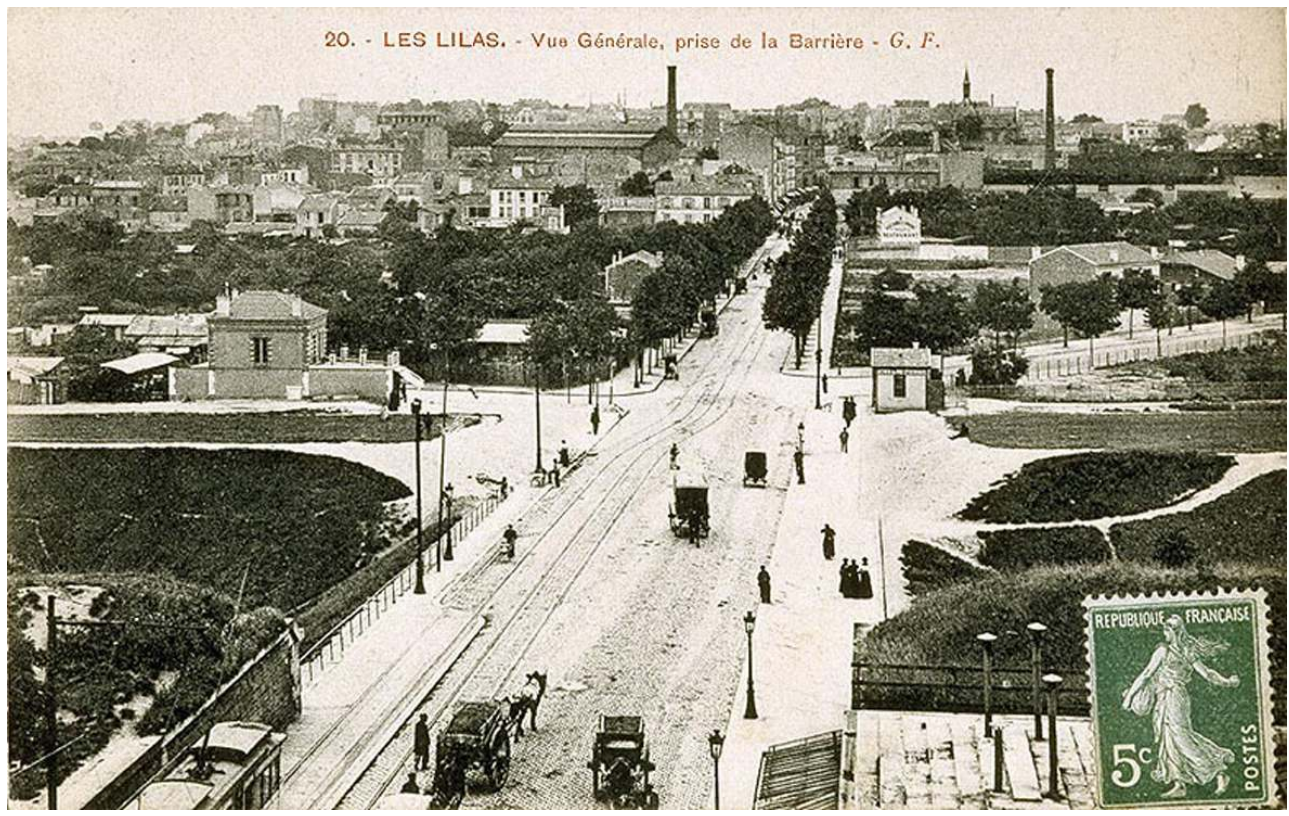

Au sud de la rue de Paris, la cheminée de l'ancien dépôt de tramways. A droite, la cheminée de l'usine Kalker (carte postale, s.d., v. 1900, AD Seine-Saint-Denis, 2Fi Les Lilas 61). Phot. Inv. L. Kruszyk (c) Inventaire général, ADAGP, 2005

Figure 2

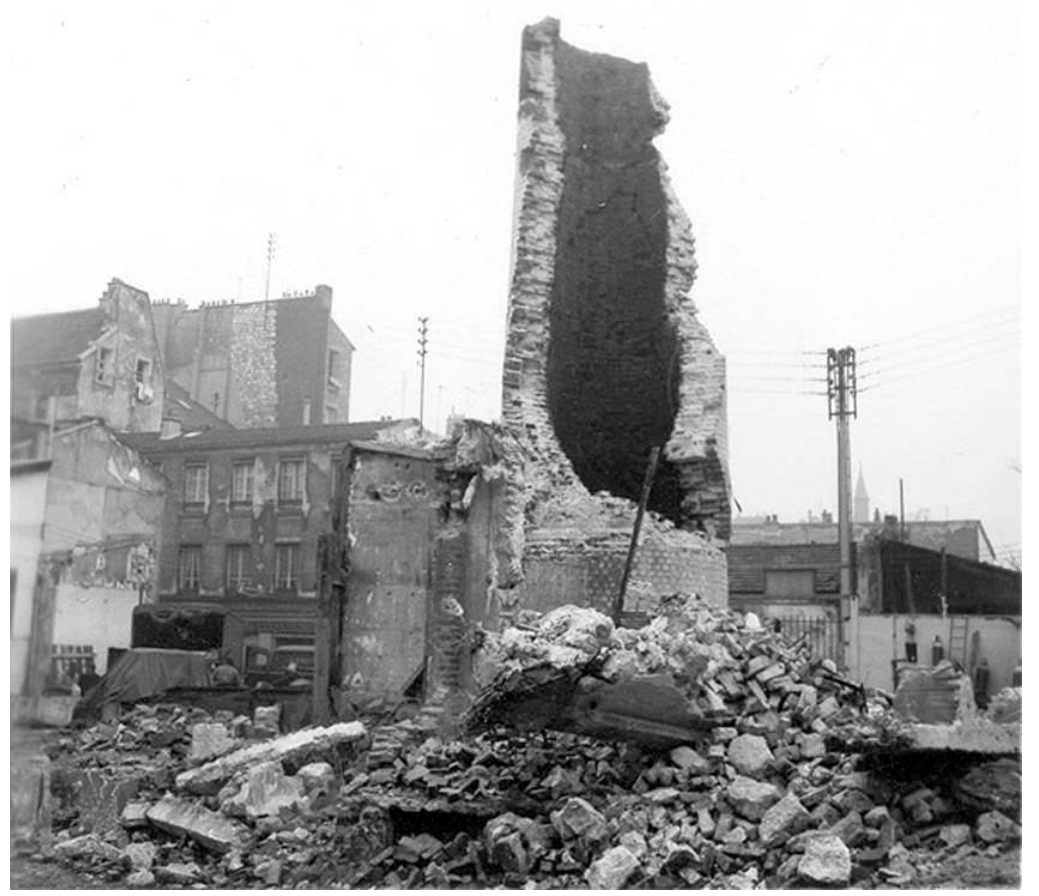

Destruction de la cheminée de l'usine Kalker, 1968 (coll. Jean Huret). Phot. Inv. L. Kruszyk (c) Inventaire général, ADAGP, 2005 
2 La liste est courte, la « reconquête » limitée. Non que les fortes emprises occupées par les activités productives et logistiques soient encore nombreuses aux Lilas : seuls demeurent les établissements Drieux-Combaluzier (ascenseurs) et le dépôt de bus Floréal ${ }^{12}$. Simplement, l'histoire du travail ne se confond pas ici avec celle de la grande industrie ${ }^{13}$. Loin de la Plaine Saint-Denis, de ses industries lourdes, notamment chimiques et métallurgiques, dévoreuses d'espace et génératrices d'emplois peu qualifiés, les Lilas, à l'image des communes de Montreuil ${ }^{14}$, de Bagnolet, du Pré-Saint-Gervais et de Pantin (au sud de l'actuelle RN3) ont accueilli à partir de la seconde moitié du XIXe siècle des activités diversifiées, en provenance notamment de l'Est parisien, des établissements de moyenne ou petite taille employant souvent une main-d'œuvre qualifiée (fig. $\mathbf{n}^{\circ} \mathbf{2}$ ).

3 Le territoire de la commune se distingue toutefois, à la lecture de la documentation rassemblée, par une forte densité de petites entreprises, par le renouvellement fréquent des activités - au sein d'un même bâtiment ou sur une même parcelle - et, en contrepoint, par la rareté des entreprises moyennes. La consultation des annuaires commerciaux ${ }^{15}$ et de l'importante série de dossiers constitués par les services préfectoraux des établissements classés dangereux, incommodes ou insalubres ${ }^{16}$, révèle que plus de 869 entreprises ${ }^{17}$ artisanales ou industrielles se sont implantées aux Lilas entre 1870 et 1980. Soit plus de 634 lieux de production, certains bâtiments - ou ensemble de bâtiments signalés à la même adresse - ayant pu accueillir jusqu'à 5 entreprises conjointement, et 9 consécutivement.

$4 \quad$ Malgré les carences de la source principale (toutes les entreprises ne s'inscrivent pas au Bottin, dont les indications sont, en outre, de précision variable), il est possible de suivre les grandes phases d'industrialisation de la commune.

\section{Les variations de la conjoncture}

5 La première phase d'industrialisation, amorcée avant la création de la commune en $1867^{18}$ , a permis l'installation d'une quarantaine d'entreprises (tableau 1). On décèle, au cours des années 1890, les conséquences de la Grande Dépression, qui suscita moins de créations que de fermetures, au sein d'un réseau de sous-traitance très sensible aux variations de la conjoncture économique. Après la forte croissance industrielle des années 1920, atteignant à l'échelle nationale près de $10 \%$ par an, et stimulant la création de plus de 100 ateliers ou usines, la commune subit la crise des années 1930. Classiquement, les Trente Glorieuses, notamment la décennie 1960-1970, marquent l'apogée de l'activité fabricante (305 entreprises). Elle précède la crise des années 1970, à l'origine d'un mouvement de désindustrialisation aujourd'hui presque achevé. L'amplitude des variations est caractéristique d'un tissu de petites et moyennes entreprises à la fois réactif aux phases de croissance et vulnérable aux crises économiques. 
Tableau 1 - Creations et femetures d'entreprises industrielles ou artisanales aux Lilas, 1870-1980

\begin{tabular}{|l|l|l|l|}
\hline & $\begin{array}{l}\text { Nombre dentreprises } \\
\text { en activité durant iacreations } \\
\text { décennie }\end{array}$ & $\begin{array}{l}\text { Fermetures } \\
\text { d'entreprises }\end{array}$ \\
\hline decennie & 38 & & \\
\hline 1870 & 63 & +40 & -15 \\
\hline 1880 & 65 & +35 & -33 \\
\hline 1890 & 73 & +49 & -43 \\
\hline 1900 & 74 & +42 & -41 \\
1910 & 161 & +112 & -25 \\
1920 & 161 & +91 & -91 \\
1930 & 219 & +107 & -49 \\
1940 & 224 & +115 & -110 \\
1950 & 305 & +134 & -55 \\
1960 & 185 & +36 & -156 \\
1970 & & & \\
\hline
\end{tabular}

Sources: Bottins du commerce (dépouill ement tous les 5 ans), dossiers des établissements classés dangerexz, incommodes ou insalubres ( $A D$ Seine-Saint-Denis).

Sources : Bottins du commerce (dépouillement tous les 5 ans), dossiers des établissements

classés dangereux, incommodes ou insalubres (AD Seine-Saint-Denis).

\section{Des activités diversifiées}

7 Il serait vain de proposer ici la liste des activités qui depuis plus d'un siècle se côtoyèrent ou se succédèrent sur le territoire de la commune. Quelques tendances peuvent être proposées, en guise de repère. A partir des années 1860, l'urbanisation et les problèmes d'exploitation concourent à la fermeture des carrières, peu nombreuses, situées au nord entre les zones d'extraction du Pré-Saint-Gervais et de Romainville. La carrière de plâtre J. Demenge cesse la dernière son activité, entre 1875 et 1880 . Une quarantaine d'entreprises, attirées « par le coût raisonnable des terrains et des baux, la patente moins élevée et les droits beaucoup plus faibles que ceux de l'octroi parisien ${ }^{19}$, s'installent à l'ouest et au centre du territoire communal, le long de voies conduisant à la rue de Paris. On retrouve les établissements de taille moyenne déjà cités, à l'importante longévité puisque détruits au cours des années 1960-1970 : les usines de caoutchouc et de celluloïd Kalker et Hemmen, le fabricant d'arômes Patrelle et, assurant le même type de production, les établissements Rozière, 8 , rue de Bagnolet (actuelle avenue du MaréchalJuin).

8 Entre 1870 et 1914, les implantations sont régulières, malgré le nombre important des fermetures au cours de la Grande Dépression. Elles confirment la domination des secteurs de la petite métallurgie, de l'habillement, du bois et du caoutchouc: 18 unités de production de caoutchouc sont créées, dont, vers 1900, la fabrique de bretelles et jarretières Boemare puis Chantalou, 15, avenue du Maréchal-Juin (détruite après inventaire en décembre 2005) ${ }^{20}$. Pour alimenter le marché parisien et le marché local, des ateliers de petite taille travaillent le bois, fabriquent des jouets et des instruments de musique, notamment des pianos, produisent et vendent de la bière, du cidre ou de l'eau 
de Seltz, façonnent le verre et les cristaux, forgent et assemblent des serrures, tournent des métaux, confectionnent des articles de maroquinerie, des boutons, des chaussures, des articles de bonneterie, de passementerie, des vêtements: en 1909, la maison de confection Ohresser, dont le magasin de vente se situe à Paris, 130-132, rue Montmartre, installe aux Lilas ses ateliers, 9, rue de Romainville ${ }^{21}$ - bâtiment transformé et agrandi au début des années 1940 par l'entreprise JO-CA pour aménager une usine de confiserie, aujourd'hui convertie en logements ${ }^{22}$ (fig. $\mathbf{n}^{\circ}$ ).

Figure 3

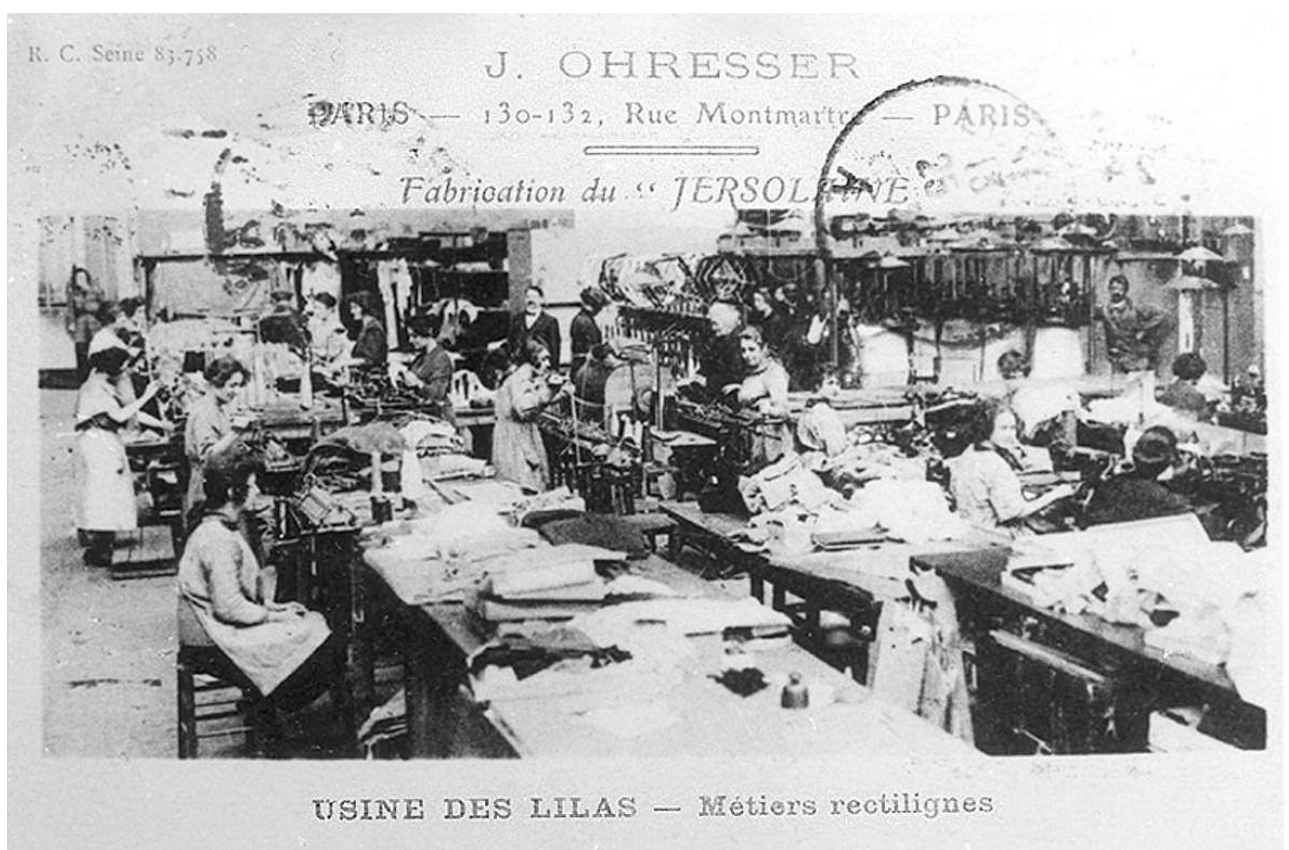

Intérieur de l'usine de confection Ohresser (repr. de carte postale, s.d., v. 1910-1920, coll. Jean Huret). Phot. Inv. L. Kruszyk

(c) Inventaire général, ADAGP, 2005

9 La croissance des années 1920, puis la reprise de la fin des années 1930 confirment les orientations des années précédentes. L'implantation le long des voies conduisant à Paris (rue de Paris, rue de Noisy-le-Sec, rues Romain-Rolland et de Romainville par l'avenue Pasteur) et à la rue de Paris (rue Henri-Barbusse, rue du Coq-Français, avenue du Maréchal-Juin et boulevard de la Liberté) est encore privilégiée. La métallurgie s'impose, avec la construction, en 1922, de l'usine de tubes sans soudure en cuivre et laiton Louis Vuillet, puis Cuprotube (25, rue Chassagnole, actuels ateliers de décors de théâtre Jipanco et salle de répétition «Lilas en Scène ») ${ }^{23}$. Les activités liées au traitement de surface des métaux - polissage, chromage, nickelage - se diffusent au sein d'ateliers installés en fond de cour. Les années 1940, 1950 et 1960 ne viennent pas remettre en cause cette tendance, malgré l'introduction de cartonneries, d'imprimeries, de laboratoires et d'ateliers de fabrication de produits pharmaceutiques (la société Ile-de-France Pharmaceutique succède, par exemple, à la fabrique de confiserie JO-CA, entre 1960 et 1965). Un dernier établissement de taille moyenne, celui de la Société industrielle de soie à coudre (acquise par l'entreprise Gütermann au cours des années 1980) est édifié en 1951 dans le quartier des Bruyères à proximité de Paris, 55-57, avenue Pasteur ${ }^{24}$. 
Tableau 2 - Évolution de l'activité par branches, 1870-1980

\begin{tabular}{|c|c|c|c|c|}
\hline & $1870-1914$ & $1914-1940$ & $1940-1960$ & $1960-1980$ \\
\hline Extraction & 1 & lo & 0 & 0 \\
\hline $\begin{array}{l}\text { Métallurgie, } \\
\text { petite métallurgie, } \\
\text { construction mécanique } \\
\text { Construction électrique, } \\
\text { mécanique de précision, } \\
\text { nstruments de musique, } \\
\text { fouets }\end{array}$ & $\int_{5}^{30}$ & 60 & 34 & 21 \\
\hline $\begin{array}{l}\text { Chimie, } \\
\text { fabrication et transformation } \\
\text { du caoutchouc }\end{array}$ & 14 & 10 & 3 & 2 \\
\hline $\begin{array}{l}\text { Testile, } \\
\text { pabillement, } \\
\text { travail du cuir }\end{array}$ & 28 & 38 & 28 & 18 \\
\hline $\begin{array}{l}\text { Verrerie, } \\
\text { céramique }\end{array}$ & 6 & 3 & 5 & 1 \\
\hline Travail du bois & 28 & 27 & 22 & 19 \\
\hline Alimentation & 9 & 10 & 2 & 2 \\
\hline Papeterie & $b$ & 2 & 4 & 3 \\
\hline Imprimerie & 2 & 5 & 4 & 1 \\
\hline $\begin{array}{l}\text { Construction } \\
\text { (dont entrepóts) }\end{array}$ & 18 & 20 & 11 & 9 \\
\hline Autres. & 7 & 18 & 5 & 3 \\
\hline
\end{tabular}

Sources: Bottins du commerce (dépouill ement tous les 5 ans).

Sources : Bottins du commerce (dépouillement tous les 5 ans).

\section{Paysages et architectures de la petite industrie}

10 L'enjeu de l'inventaire consiste, par la suite, à identifier la nature des réponses architecturales aux secousses de la conjoncture et aux contraintes de la production, en fonction notamment de la topographie, du parcellaire et de l'histoire particulière de chaque entreprise. D'où la nécessité de considérer, dans la continuité d'une démarche inaugurée sur le territoire de Montreuil ${ }^{25}$, le bâti et les vestiges de la petite industrie et de l'artisanat (on songe, dans ce dernier cas, aux ateliers employant une ou deux personnes).

Le repérage îlot par îlot et, à l'intérieur de chaque îlot, parcelle par parcelle, a permis de constater la destruction de 248 sites, sur 634 documentés; 53 adresses d'entreprises disparues correspondent à 20 immeubles et 33 maisons ayant vraisemblablement accueilli une activité en rez-de-chaussée, en sous-sol ou en chambre, hypothèse qu'il ne nous a pas été possible de vérifier; 119 sites, souvent de très petite taille, ont éventuellement accueilli une activité de production, sans que l'implantation ou l'architecture des bâtiments ne permettent de l'affirmer avec certitude ; 88 établissements mentionnés par les sources, sans adresse, ou celle-ci demeurant imprécise en raison notamment des modifications de la numérotation, n'ont pu être localisés ; 34 sites en cœur d'îlot nous sont demeurés inaccessibles. Enfin, 110 sites dont la fonction de production ou d'entreposage est attestée avec certitude ont été repérés (18 ayant été identifiés grâce à la seule enquête de terrain) (fig. $\left.n^{\circ} 4\right)$. 
Figure 4

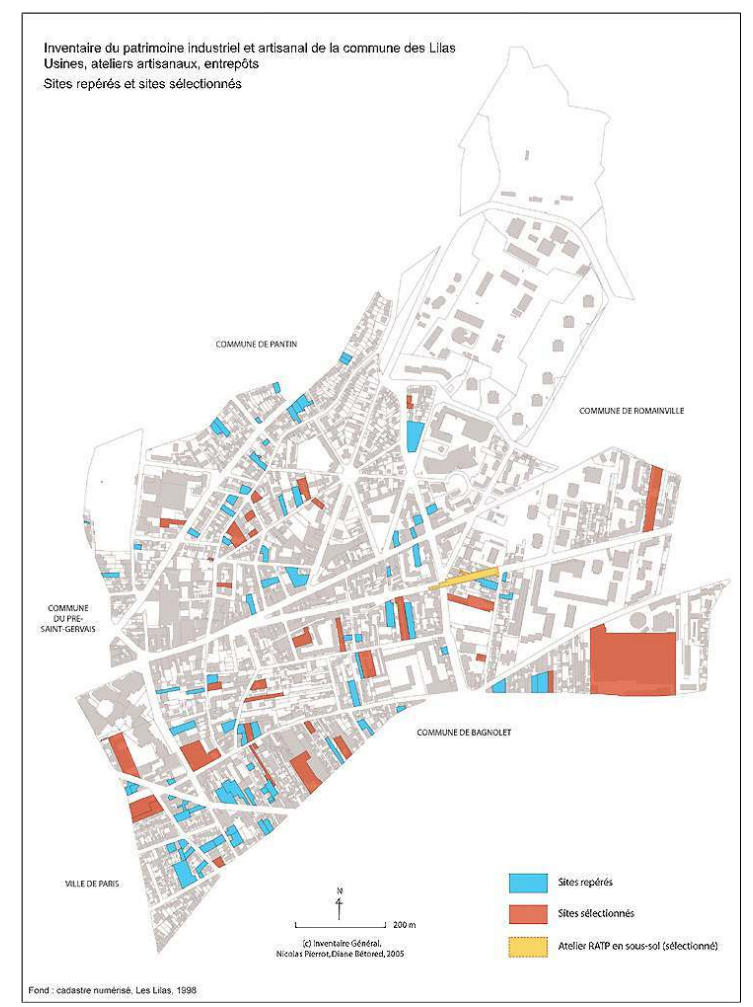

Patrimoine industriel et artisanal de la commune des Lilas. Carte des sites repérés et sélectionnés (c) Inventaire général, Diane Bétored, ADAGP, 2005

Le nombre important d'éléments dénaturés ainsi que la possibilité d'identifier plusieurs groupes de récurrence formelle autorisait de procéder à la sélection de bâtiments ou ensemble de bâtiments représentatifs ou remarquables : 32 sites ont été retenus ${ }^{26}$. Ils ont fait l'objet d'une étude historique et architecturale, mobilisant les fonds d'archives départementales (établissements classés, matrices cadastrales) et municipales (établissements classés, permis de construire), ainsi que plusieurs témoignages oraux. Les établissements Kalker, quoique détruits, ont également fait l'objet d'une étude, en raison de leur importance historique.

Afin de souligner les grandes caractéristiques du bâti industriel et artisanal de la commune, les édifices repérés et sélectionnés peuvent être répartis en familles, en fonction de leur datation, de leur implantation (taille et forme des parcelles, présence de cours, situation relative des bâtiments de production, des espaces de stockage, des bureaux et des logements), et de leur structure (étages, organisation de la production verticale ou horizontale, matériaux, type de charpente et de couverture, décor).

Reflétant approximativement la chronologie de l'industrialisation, $14 \%$ des édifices repérés peuvent être datés, pour les parties les plus anciennes, entre 1870 et 1914, $36 \%$ entre 1914 et 1940, 34 \% entre 1940 et 1960, et $16 \%$ entre 1960-1980. Ceux de la première génération sont rares, les traces en sont discrètes, côtoyant les constructions plus récentes de la même entreprise ou d'une nouvelle entreprise installée sur le même site. L'ensemble des secteurs industriels est représenté, reflétant la hiérarchie suggérée par le tableau 2. 
De l'approche structurelle (morphologie et distribution), on retiendra surtout l'opportunité de distinguer plusieurs catégories au sein du groupe majoritaire des bâtiments relevant de la petite industrie, comprenant 1 à 5 bâtiments de petite taille ( $80 \%$ des sites repérés, soit 82 unités de production et 6 entrepôts). Première catégorie : le fait majeur est l'implantation à l'arrière de la parcelle, dont on détaillera la variété des formes. On distinguera ensuite les bâtiments alignés sur la rue, offrant parfois un décor. Une dernière catégorie est réservée aux sites de taille moyenne, soit 18 usines souvent dénaturées ou partiellement détruites, et 4 entrepôts ( $20 \%$ des sites repérés). Le dépôt de bus Floréal et l'atelier RATP de réparation du matériel roulant de la ligne 11 - en sous-sol constituent deux exceptions. Ils suggèrent la forte présence de l'entreprise sur le territoire et dans l'économie de la commune.

\section{Au fond des cours, le long des sentes : des ateliers dissimulés}

Les importants regroupements de parcelles sont rares, traduisant peut-être, outre les contraintes liées à la topographie, une tendance longue de la politique municipale, hostile aux fortes emprises industrielles et logistiques. Quelques exceptions toutefois, au sud et à l'est de la commune: l'usine d'équipement aéronautique «Etudes, Réalisations, Accessoires mécaniques » (ERAM), installée 153, rue de Noisy-le-Sec entre 1955 et 1960 (actuellement usine d'ascenseurs Drieux-Combaluzier) ${ }^{27}$, le dépôt de bus Floréal, dont les premiers bâtiments furent édifiés avant $1900^{28}$, et l'usine de la Compagnie du Ronéo ${ }^{29}$ dont subsistent deux bâtiments habillés de façades-miroirs en vue d'accueillir un ensemble de bureaux de l'entreprise Nokia.

Plus des deux tiers des établissements repérés, relevant de l'artisanat ou de la petite industrie, possèdent une cour. Espace de chargement, de livraison, de stockage, et de distribution entre les bâtiments, elle est tracée en fonction des besoins de l'activité et de la forme des parcelles. Les ateliers de la petite industrie présentent ainsi trois types d'implantations.

L'activité peut avoir élu domicile en fond de cour, dans un atelier simple, à pans de bois, de fer ou de béton hourdés de brique, en appentis ou couvert d'un toit-terrasse. L'atelier peut également être implanté au fond d'une parcelle en lanière, héritée de l'activité agricole, et s'inscrire dans sa longueur; il est alors couvert d'un toit à longs pans, et présente son pignon sur la cour, ouvrant parfois sur la rue, mais souvent masqué par un pavillon. L'ancien atelier de serrurerie et ferronnerie Albert Brelot, attesté en 1925 au 40, rue du Tapis-Vert, en constitue l'exemple le mieux conservé ${ }^{30}$. Un immeuble peut également dissimuler sur la rue l'espace de fabrication. On songe à l'usine de boîtes métalliques Houpermann (175, rue de Noisy-le-Sec), qui présente, derrière un immeuble élevé vers 1900, la plus ancienne halle à pans de bois hourdés de briques, conservée sur le territoire de la commune ${ }^{31}$. Construite entre 1905 et 1910, il n'en subsiste plus aujourd'hui que la structure et la charpente en bois, à l'issue d'une importante campagne de réhabilitation en logements (fig. $n^{\circ} 5$ ). 
Figure 5

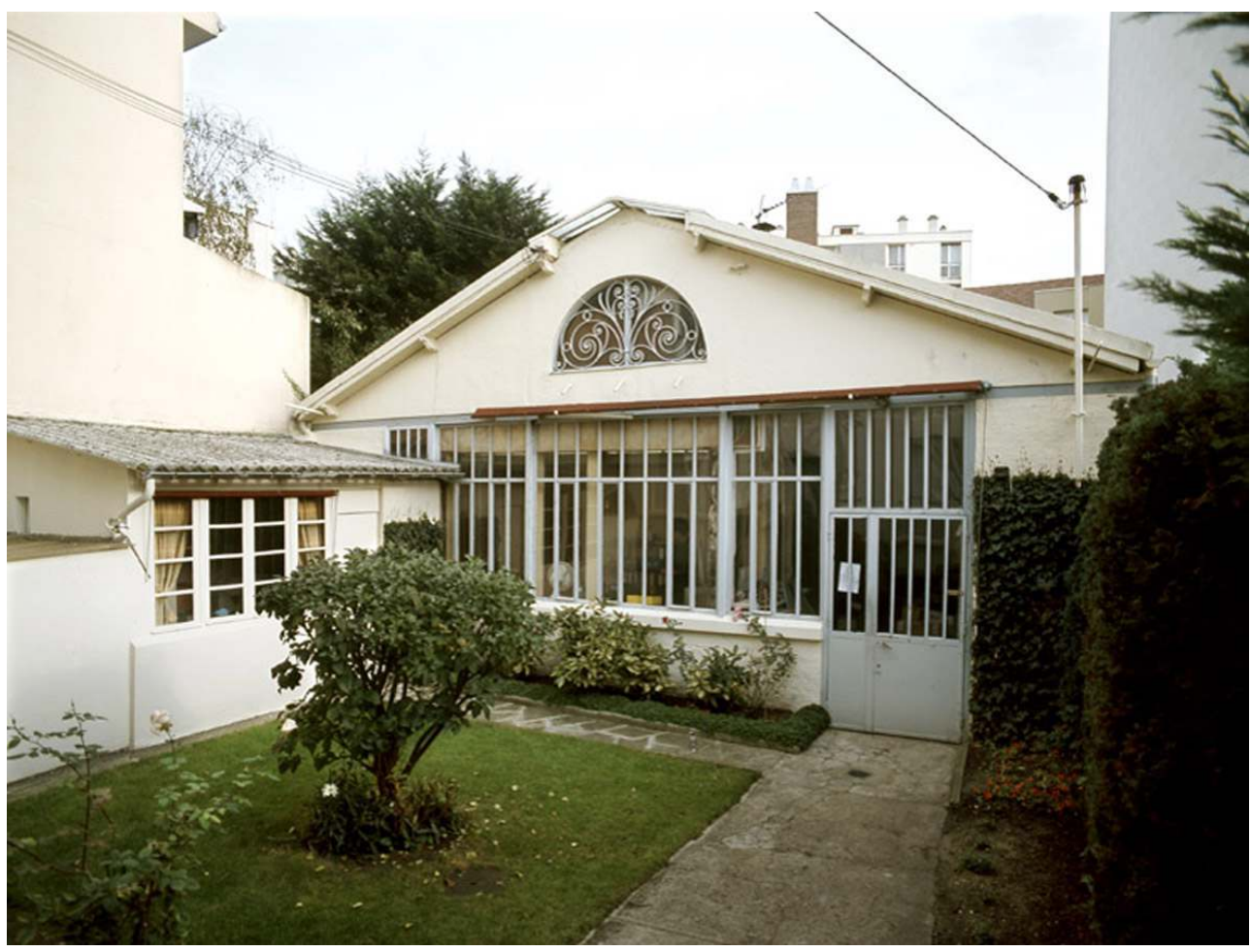

Pignon de l'ancien atelier de serrurerie Brelot, 40, rue du Tapis-Vert. Phot. Inv. L. Kruszyk

(C) Inventaire général, ADAGP, 2005

19 D'autres ateliers occupent le flanc de parcelles en lanières plus étroites. L'usine, de plan rectangulaire allongé, bordée d'un passage ou d'une cour, est élevée sur plusieurs étages lorsque l'activité, le plus souvent d'assemblage ou de confection, nécessite peu d'installations pondéreuses: c'était le cas de la fabrique de bretelles et jarretières en caoutchouc Boemare puis Chantalou (15, rue du Maréchal-Juin) construite vers $1900^{32}$. Avant sa destruction en 2005, il s'agissait du plus ancien bâtiment industriel de la commune. L'étage était vraisemblablement dédié aux activités les plus minutieuses: ouvertes dans un mur à pans de bois hourdés de brique - structure légère - de larges baies horizontales éclairent l'intérieur de l'atelier. Autre exemple, les machines de la bonneterie Goldschmidt étaient installées dès le milieu des années 1930 au rez-dechaussée $^{33}$, alors que les deux étages du bâtiment étaient affectés aux opérations faiblement mécanisées. Encore en place, le pavillon construit à l'entrée du site (22, rue Romain-Rolland), abritait le logement patronal et le bureau de l'entreprise ${ }^{34}$ (fig. $\mathbf{n}^{\circ} \mathbf{6}$ ). 


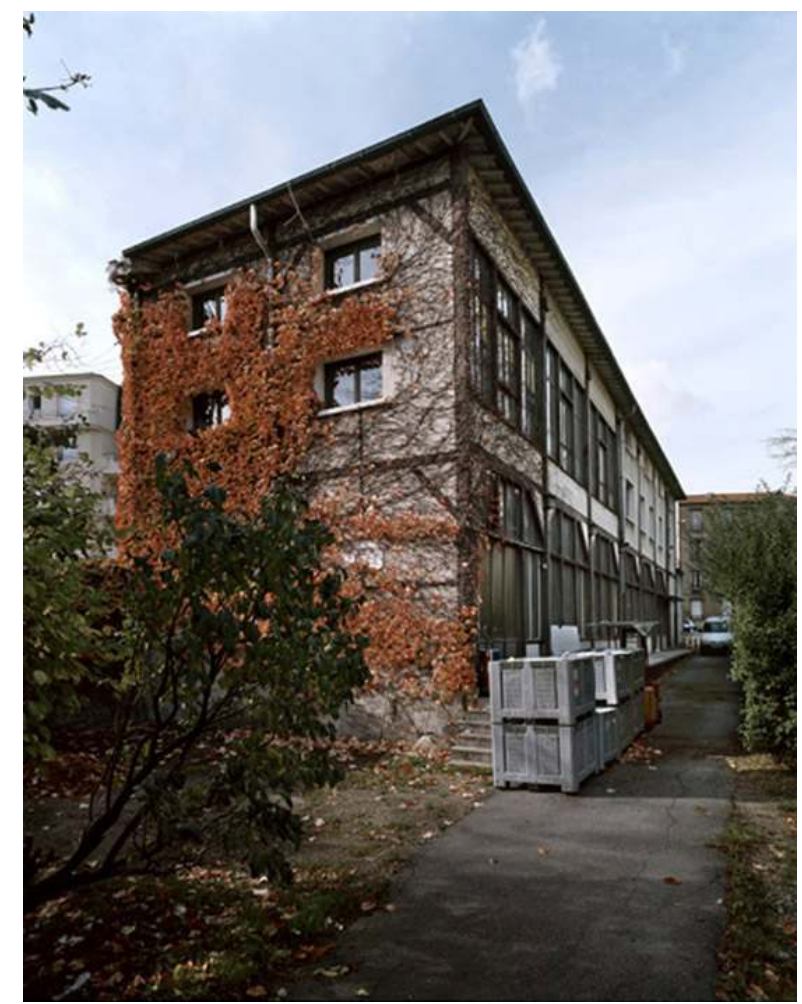

Ancienne usine de bretelles et jarretières en caoutchouc Bœmare, puis Chantalou, 15, avenue du Maréchal-Juin (détruit après inventaire). Phot. Inv. L. Kruszyk

(C) Inventaire général, ADAGP, 2005

Troisième catégorie, les bâtiments de production occupant les trois flancs d'une parcelle en lanière, dessinant une cour centrale. S'y déploient des activités nécessitant plusieurs ateliers distincts. Il s'agit parfois du stade final d'une évolution dont la lecture du bâti suggère les vicissitudes ${ }^{35}$. Les fonderies, abritant des équipements de forte charge, sont en rez-de-chaussée. On peut mentionner la fonderie d'aluminium Mathieu, 35, rue du TapisVert, construite en 1939, ou la fonderie de cuivre, de bronze et de laiton Piattino, 28, rue Romain-Rolland, dont l'organisation de la production est connue grâce au témoignage de M. Jean-Pierre Piattino, fils de l'entrepreneur Marius-Jean Piattino ${ }^{36}$ (1914-1969) (fig. n ${ }^{\circ} 7$ ). 


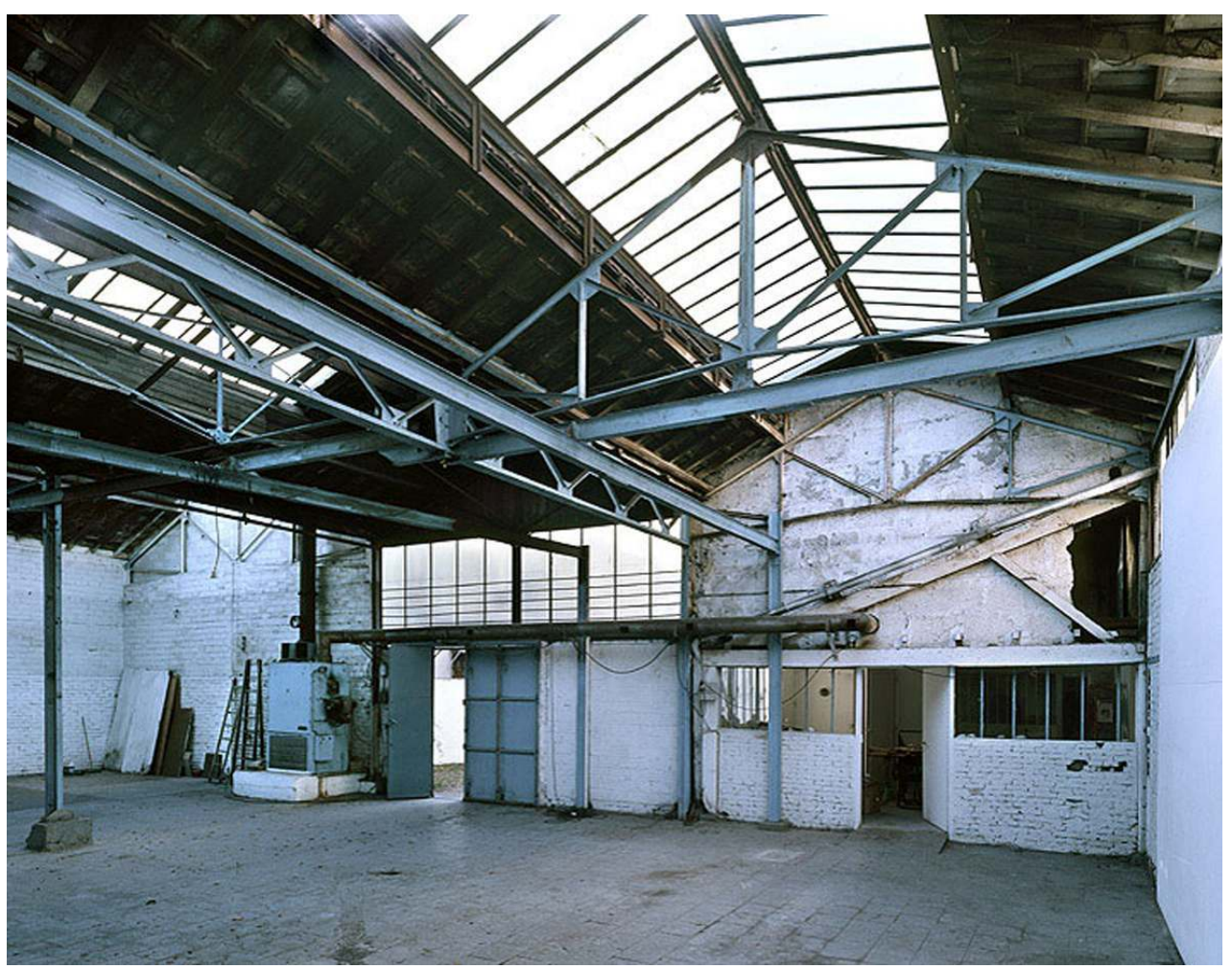

Intérieur de l'ancienne fonderie de cuivre, bronze et laiton Piattino, 28, rue Romain-Rolland. Phot. Inv. L. Kruszyk

(c) Inventaire général, ADAGP, 2005

21 Le sable et la terre argileuse, destinés à la confection des moules, étaient déposés sur le sol de l'atelier; une fois les moules et les noyaux confectionnés, un passage à l'étuve permettait leur solidification; les poches de coulée de $150 \mathrm{~kg}$ étaient guidées, depuis les cubilots, par un treuil relié au rail encore présent au plafond de l'atelier ${ }^{37}$. Pour accueillir ces opérations, Marius-Jean Piattino avait fait agrandir, entre 1947 et 1949, l'ancienne usine Mathieu, construite à cette adresse entre 1930 et 1939, avant son déménagement ${ }^{38}$ 35, rue du Tapis-Vert ${ }^{39}$. L'établissement Piattino comprend ainsi une grande halle de fonderie et d'étuvage en fond de parcelle et, orthogonalement, une halle de sablage, un magasin à châssis et un magasin d'expédition, soit trois halles à pans de fer hourdés de briques surmontées d'une charpente en bois pour la plus ancienne, métalliques à fermes triangulées pour les plus récentes, coiffées de lanterneaux vitrés pour l'éclairage et l'aération. Les deux ailes en retour (magasin aux moules, bureau et vestiaires au nord, en parpaing de mâchefer, bâtiment d'habitation au sud), formant un ensemble en U, sont héritées des sociétés précédemment installées sur le site depuis 1900-1905: les entreprises de peinture et vitrerie Munier, Souverain, puis Guimberteau, et la première fonderie $M^{2}$ athieu ${ }^{40}$. Inversement, la présence d'un étage carré est significative d'une activité d'assemblage, souvent manuelle ou faiblement mécanisée : on songe au bâtiment principal, en maçonnerie de brique, de l'ancienne manufacture de jouets Dreyfuss et Riès, 81 , rue Romain-Rolland, construit vers $1925^{41}$. Implanté sur le flanc oriental de la parcelle, il comporte un étage carré percé de larges baies horizontales inspirées de l'architecture industrielle américaine du début du XXe siècle. A l'ouest, les bâtiments en rez-dechaussée semblent avoir été construits au gré de l'agrandissement de l'usine, sans 
remettre en cause le principe rationnel de la distribution autour de la cour qui s'ouvre sur la rue Romain-Rolland. Mais une nouvelle fois, à l'adresse principale de l'entreprise, 44, boulevard de la Liberté, les bâtiments de production sont masqués par un immeuble (fig. $\left.n^{\circ} 8\right)$.

Figure 8

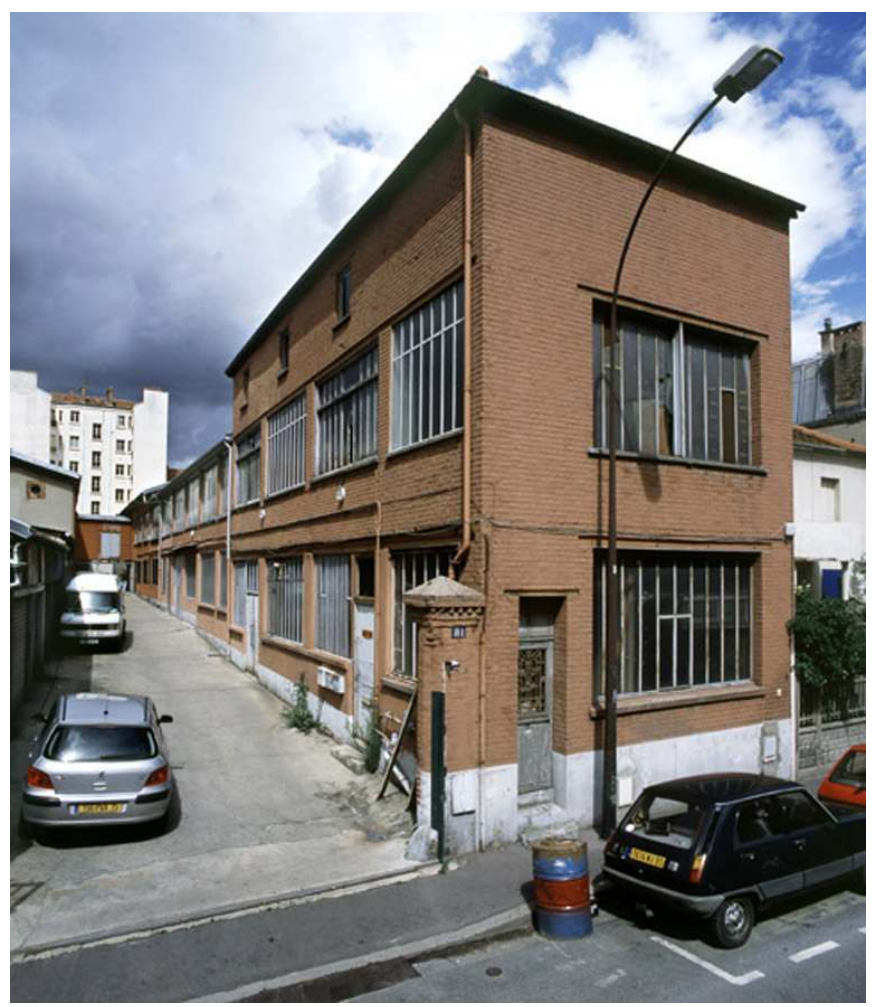

Vue de l'ancienne usine de jouets Dreyfus et Riès, 81, rue Romain-Rolland. Phot. Inv. L. Kruszyk (c) Inventaire général, ADAGP, 2004

Enfin, cas exceptionnel sur ce territoire, le bâtiment peut occuper la totalité d'une parcelle en lanière. L'usine de teinturerie Joannart, puis Goyard, Lallier et Cie, 36, rue de Romainville, s'est développée entre 1908 et 1922 le long du passage Félix-Houdart, sur une longueur de 102 mètres et une largeur de 5 mètres $^{42}$. L'édifice en maçonnerie de pierre calcaire, puis de brique, puis à pans de fer hourdés de brique, consiste en un long rez-dechaussée. Plus onéreuse eût été la construction d'un étage, alors que le process de production linéaire est parfaitement adapté à l'activité : chaufferie, atelier de teinture, apprêt, pliage. Il était préférable, en outre, de faire reposer les cuves sur le sol. En 1922, occupant déjà l'ensemble de l'espace disponible, l'entreprise est contrainte, afin d'aménager une nouvelle salle de pliage, d'élever un étage carré au-dessus d'une salle d'apprêt, à l'extrémité sud de la parcelle ${ }^{43}$. Malgré l'évolution de son enveloppe bâtie et de son emprise au sol, l'usine demeure discrète sur la rue de Romainville, n'affichant aucunement sa fonction de production ${ }^{44}$ (fig. $\mathbf{n}^{\circ}$ 9). 
Figure 9

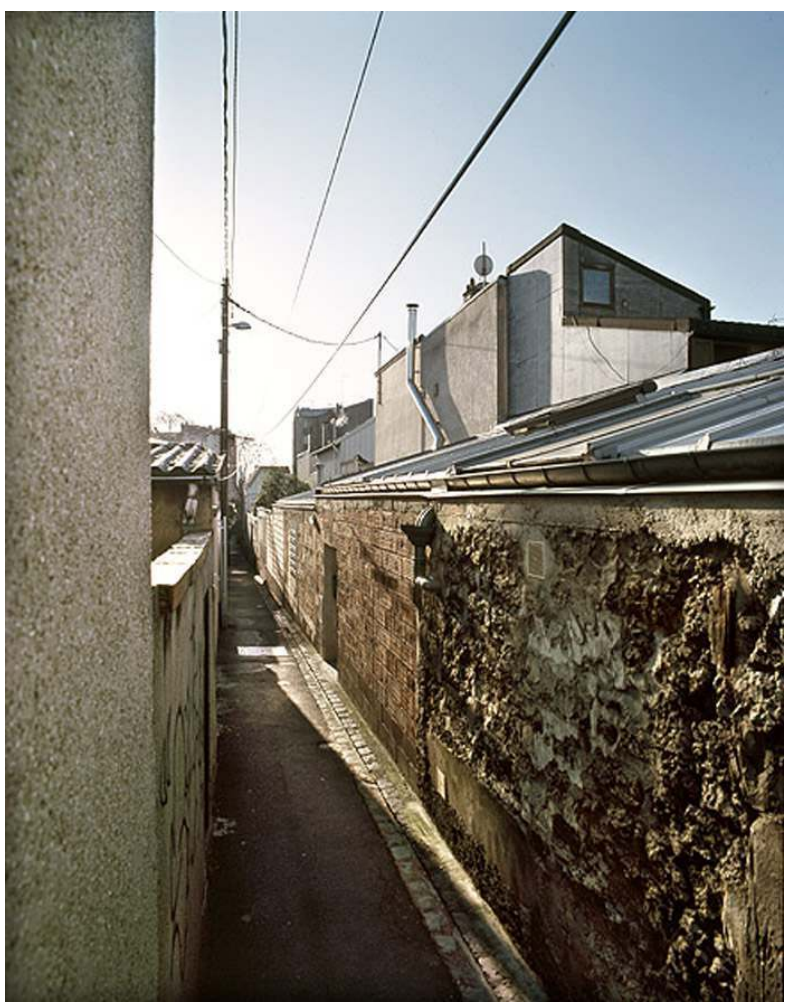

Vue de l'ancienne teinturerie Joannart, puis Goyard, Lallier et Cie, passage Félix-Houdart. Phot. Inv. L. Kruszyk

(c) Inventaire général, ADAGP, 2005

\section{Sur la rue : modestie de l'affichage}

L'indifférenciation des enveloppes bâties, n'autorisant que rarement la lecture des fonctions industrielles, est quelquefois tempérée par le choix d'afficher sur la rue, par un décor, l'identité productive ou commerciale de l'entreprise. Cette caractéristique, qui concerne un quart des bâtiments repérés, dissimule une variété de formules dont il convient de rendre compte par quelques exemples.

Les magasins de stockage et de vente des établissements Danzelle, Mairignac et Robineau ${ }^{45}$, construits en pan de béton hourdé de brique (enduit) entre 1925 et $1951^{46}$, présentent à l'angle de la rue Chassagnole et de la rue des Villegrange une façade ordonnancée, sur deux étages, l'axe de symétrie étant formé par le pan coupé marquant l'angle de la rue. Point de décor, à l'exception des motifs en ferronnerie de la porte principale, surmontée d'un entablement qui portait vraisemblablement le nom de l'entreprise. Le bâtiment dissimule un ensemble d'ateliers, en cœur d'îlot, affectés à la fabrication de boutons métalliques et autres $"$ articles de Paris $»^{47}$, employant 80 ouvriers et 20 ouvrières et employées en 1951. Les ateliers de « confection en gros pour dame $»^{48}$ Léon Ohresser, dont le magasin de vente était situé à Paris, 130, boulevard Montmartre ${ }^{49}$, affichent depuis 1909 leur présence sur la rue de Romainville, $n^{\circ} 7$ à 11 . Le portail monumental est surmonté d'un entablement portant le nom de l'entreprise » JO-CA », fabrique de bonbons installée sur les lieux vers $1945^{50}$ (fig. $\mathbf{n}^{\circ}$ 10). Ce dernier est coiffé d'une corniche de brique en dents d'engrenage et d'un chaperon de tuile. L'utilisation d'un rocaillage de meulière et 
l'ajout d'une frise en brique témoignent d'un soin particulier, reflet de l'architecture civile de la même époque. Une véranda, discrète mais remarquable par ses armatures en bois, orne la façade de la fabrique de limes Mariot-Duponchel installée 11 bis ${ }^{51}$, rue du Coq-Français vers $1925^{52}$. Cet élément de décor a été conservé malgré la succession de 6 entreprises sur le site et la reconversion des bâtiments, entre 1999 et 2002, en salle de spectacle.

Figure 10

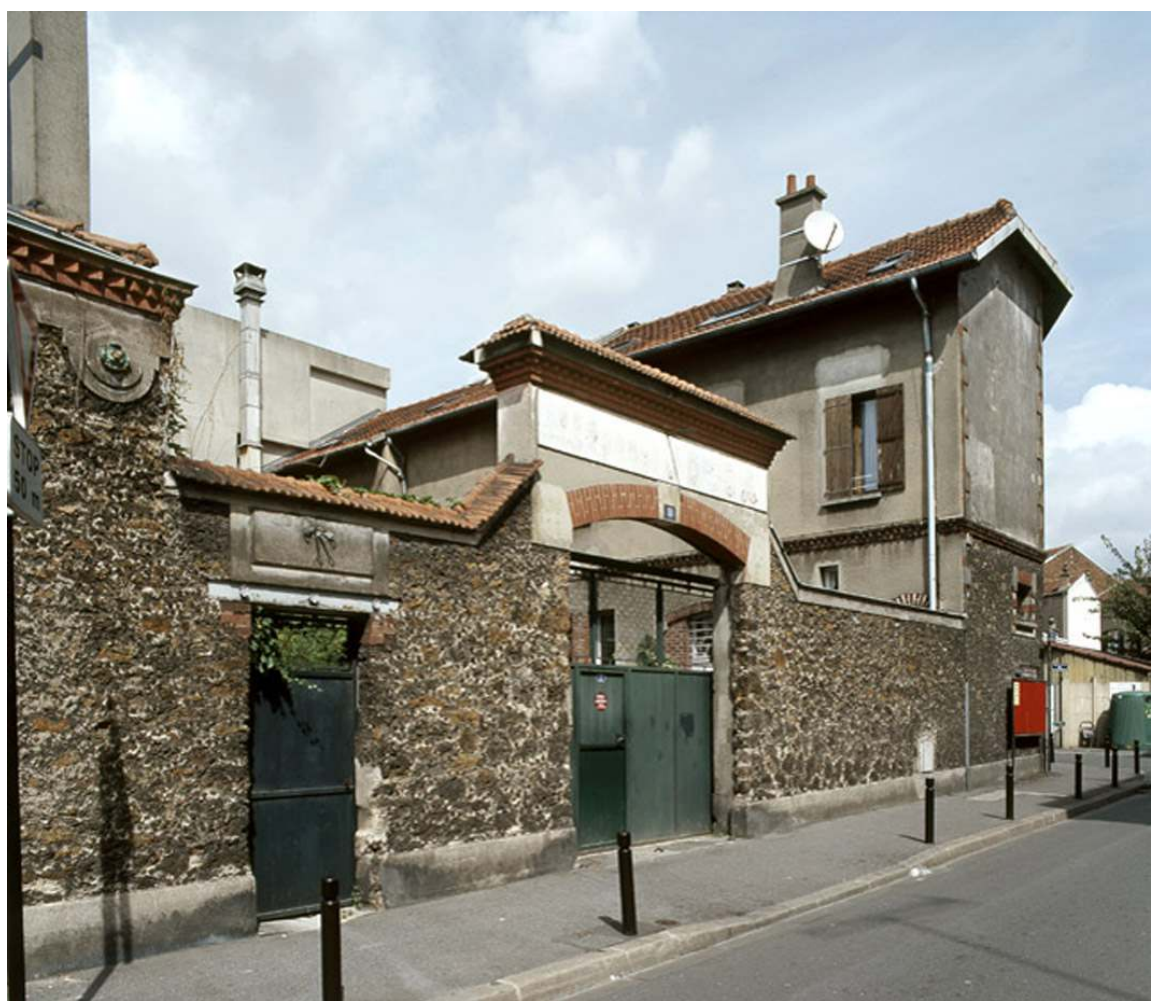

Portail de l'ancienne usine de confection Ohresser, puis confiserie JO-CA, 7-11, rue de Romainville. Phot. Inv. L. Kruszyk

(C) Inventaire général, ADAGP, 2004

Si le fronton et les modillons ornant la façade de l'entreprise TAC - Toutes les Applications de la Chaudronnerie - sise au 175, rue de Noisy-le-Sec ${ }^{53}$, marquent une volonté d'ornement et de lisibilité sur la rue, le geste demeure limité, le résultat sobre. Il n'en fut pas de même pour le dépôt de l'entreprise de terrassements et travaux publics Dupuydenus, 13, rue Raymonde-Salez, élevé en 1924 pour abriter divers véhicules de chantier ${ }^{54}$ (détruit après inventaire $)^{55}$. Simple halle à structure métallique hourdée de brique, couverte d'une charpente métallique et d'un toit à longs pans coiffé d'un lanterneau, elle présentait sur la rue une façade-pignon monumentale rythmée d'imposants pilastres cannelés. Sur le fronton, une table portait le nom de la société (fig. $\left.\mathbf{n}^{\circ} \mathbf{1 1}\right)$. 


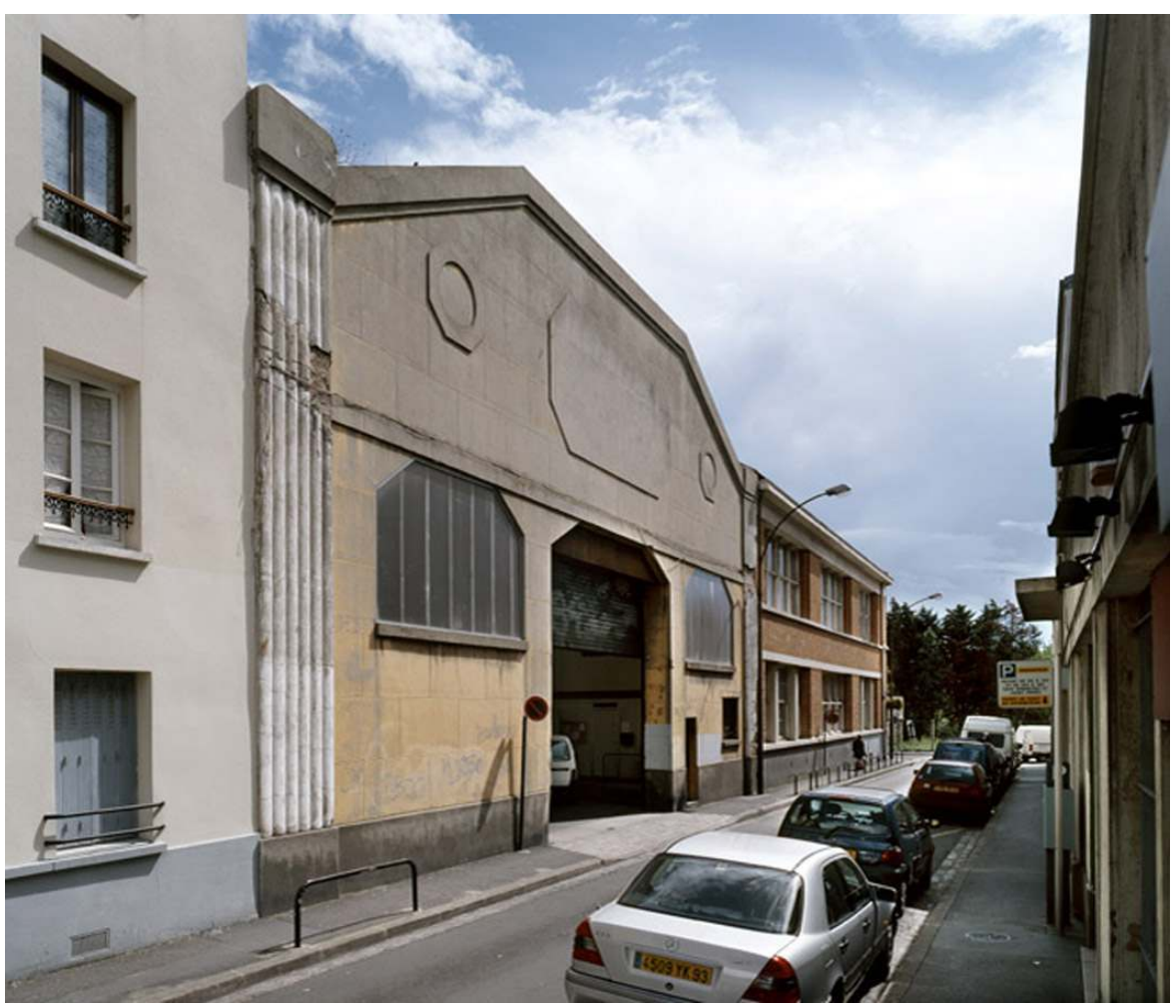

Façade de l'ancien entrepôt Dupuydenus, 13, rue Raymonde-Salez (détruit après inventaire). Phot. Inv. L. Kruszyk

(c) Inventaire général, ADAGP, 2004

\section{Les usines}

La destruction des usines Kalker, Patrelle, Rozière ou Hemmen (dont subsiste le pavillon de l'entrepreneur) ne doit pas dissimuler la persistance, sur le territoire communal, de quelques établissements de taille moyenne (18, soit $20 \%$ des sites repérés), tranchant avec le tissu d'ateliers et de petits édifices, caractéristique du paysage lilasien. L'une de ces usines demeure en activité : on songe à l'entreprise Drieux-Combaluzier (fabrication d'ascenseurs), implantée au 153, rue de Noisy-le-Sec au cours des années 1980, qui a succédé à l'équipementier aéronautique ERAM, installé entre 1955 et $1960^{56}$. L'usine de meubles pour bureau de la Compagnie du Ronéo (83, boulevard du Général-Leclercq-deHauteclocque), subsiste dans sa partie nord ${ }^{57}$. Les ateliers couverts en sheds, au sud du boulevard, ont été détruits en $1982^{58}$. Les deux bâtiments du début des années 1930, aujourd'hui convertis en immeubles de bureaux, n'ont conservé que leur structure poteaux-poutres en béton armé, des murs-rideaux en verre ayant remplacé les parois de brique. Le site est actuellement occupé par l'entreprise Nokia.

Deux ensembles se distinguent toutefois par l'intérêt et la conservation de leur architecture : la fabrique de tubes sans soudure en cuivre et laiton Louis Vulliet, puis Cuprotube, édifiée vers 1922 au 25, rue Chassagnole, et l'usine de la Société industrielle de soie à coudre, édifiée au 55-59, avenue Pasteur en 1951, acquise au cours des années 1980 par l'entreprise Gütermann. La première, de taille moyenne, diffère des ateliers, 
implantés en fond de parcelle et masqués sur la rue, formant l'essentiel du tissu industriel et artisanal de la commune ${ }^{59}$ (fig. $\mathbf{n}^{\circ} \mathbf{1 2}$ ).

Figure 12

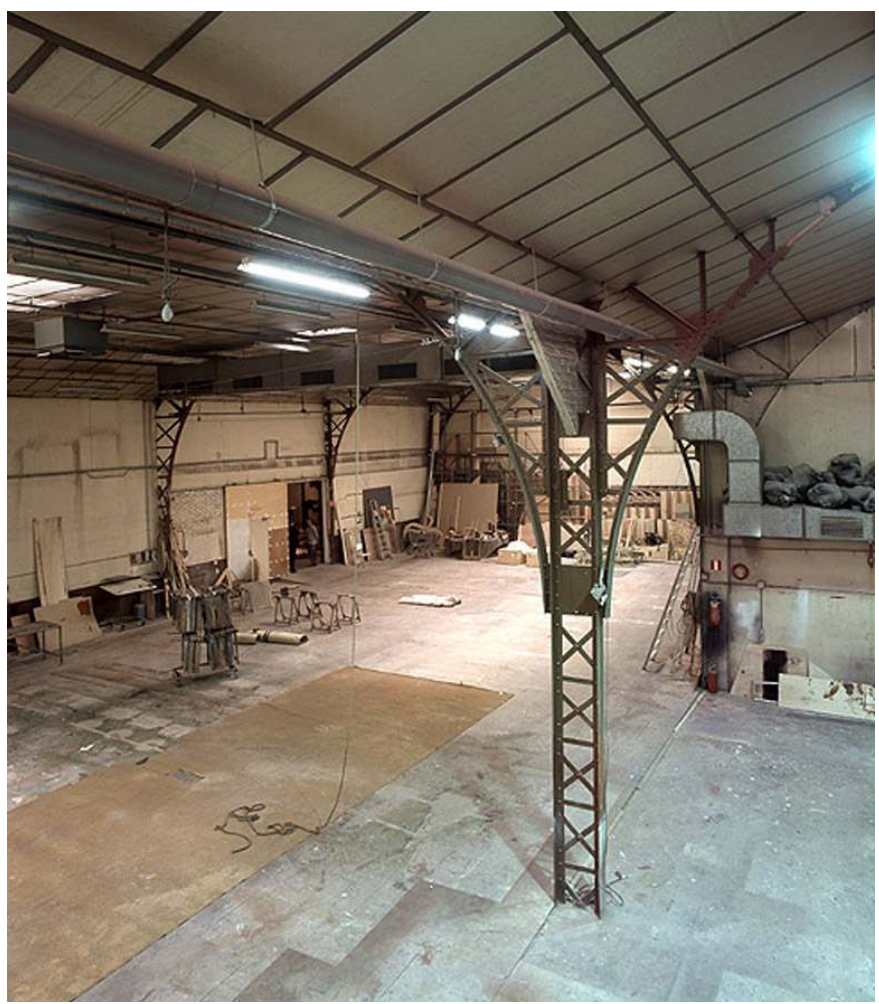

Charpente métallique, dérivée du type De Dion, de l'ancienne usine de transformation des métaux Louis Vulliet puis Cuprotube, 25, rue Chassagnole. Phot. Inv. L. Kruszyk

(C) Inventaire général, ADAGP, 2005

Elle se distingue surtout par les charpentes de ses trois halles accolées, élevées en $1922^{60}$. L'emploi de charpentes métalliques à tirants eût encombré le volume intérieur des ateliers et du magasin (au nord), au sein desquels de longues pièces de métal devaient être déplacées. L'architecte choisit d'adopter le principe de la charpente De Dion, élaboré en 1878 pour la galerie des machines de l'Exposition universelle. Les supports et les arbalétriers sont associés, formant ainsi des poutres triangulées en arc de cercle. Les tirants sont éliminés : les arcs exercent une poussée à la base des poteaux scellés dans les fondations ${ }^{61}$.

C'est également par le système de couvrement de ses trois halles accolées (occupant plus de $1000 \mathrm{~m}^{2}$ ) que se distingue l'usine de la Société industrielle de soie à coudre, construite entre $1948^{62}$ et $1950^{63}$. La toiture de chaque halle est suspendue par trois arceaux de béton armé inscrits dans la largeur du bâtiment. Trois poutres horizontales en béton armé, reposant sur six poteaux verticaux, jouent le rôle de longerons. L'architecte Aloïs R. Verrey semble avoir adapté ici la technique du pont suspendu du bâtiment industriel afin de dégager l'espace intérieur (fig. $\mathbf{n}^{\circ} \mathbf{1 3}$ ). 


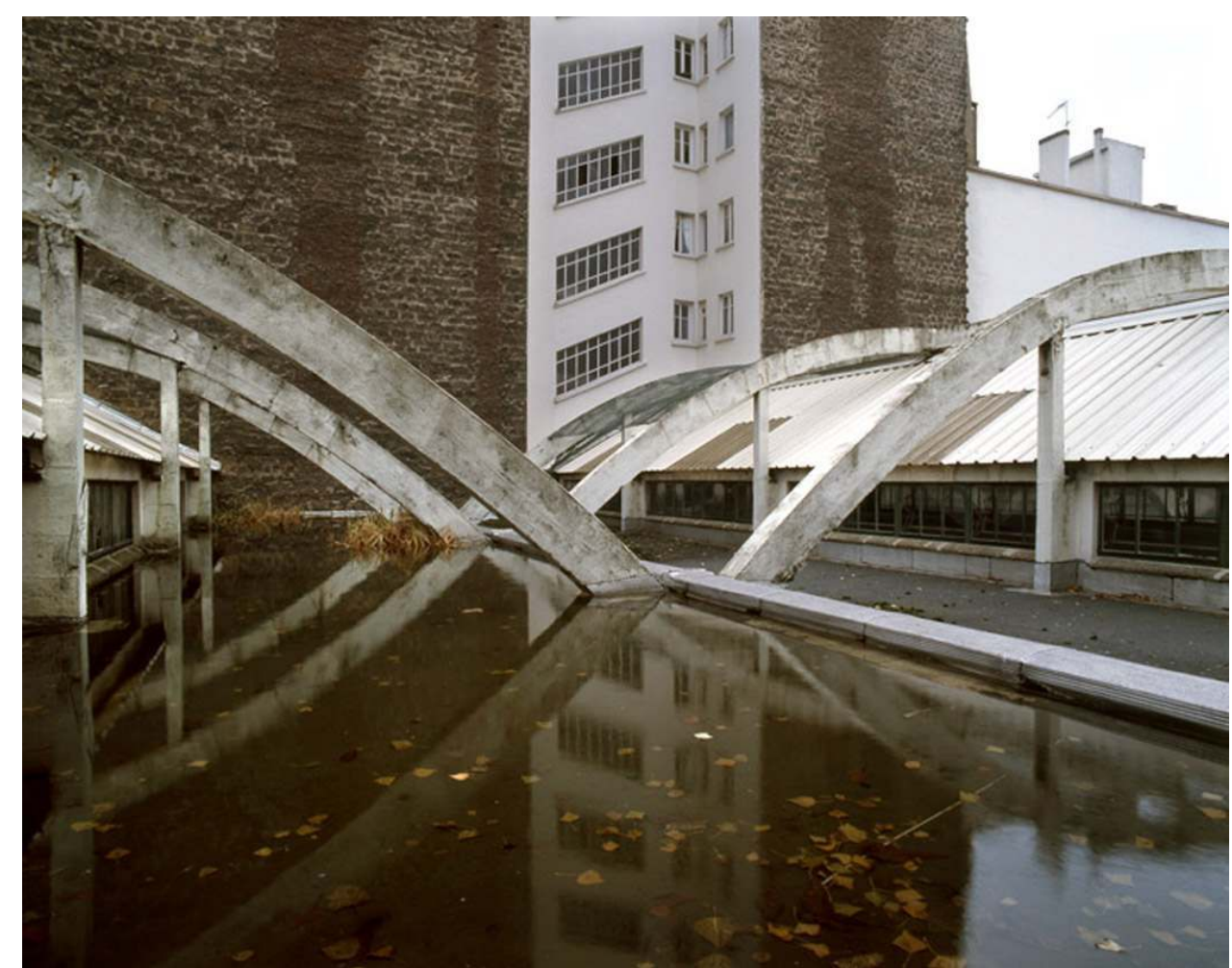

Ancienne usine de la Société industrielle de soie à coudre, puis Gütermann, 55-59, avenue Pasteur. La toiture de chaque halle est suspendue par trois arceaux en béton armé inscrits dans la largeur du bâtiment. Phot. Inv. L. Kruszyk

(c) Inventaire général, ADAGP, 2005

Il ne saurait être question de cantonner l'enquête aux limites administratives d'une seule commune : étudier l'inscription du travail industriel et son expression architecturale démonstrative ou modeste - dans une ville en mouvement, accompagner les reconversions programmées ou spontanées exige de considérer l'ensemble des communes limitrophes de la capitale, à l'Est dans un premier temps, depuis Montreuil jusqu'à Pantin ${ }^{64}$, et de poursuivre les investigations dans les arrondissements parisiens ${ }^{65}$. Ce travail, qui doit s'accompagner d'une collecte - déjà bien tardive - des archives d'entreprise et des témoignages oraux du travail peut, au terme du parcours, contribuer à l'identification d'éléments remarquables du patrimoine communal au titre de l'article L 123-1-7 du Code de l'Urbanisme, dans le cadre de l'élaboration des PLU. La difficulté, ici encore, réside dans l'articulation du temps de la recherche avec celui de l'action sur le territoire, en faveur d'un patrimoine que l'on sait fragile. 


\section{NOTES}

1. Cette contribution s'appuie sur une enquête de l'Inventaire général, réalisée en 2004-2005 avec le concours de Mathieu Chambrion, dans le cadre du protocole de décentralisation culturelle signé par le Conseil général de la Seine-Saint-Denis et le ministère de la Culture et de la Communication. Il s'agit de la version augmentée et annotée du chapitre consacré au "patrimoine industriel et artisanal », dans : LABORDE, Marie-Françoise, HERON, Claude, LOHR, Evelyne et al. Contribution au diagnostic du patrimoine de la commune des Lilas. Pantin: Bureau du Patrimoine de la Seine-Saint-Denis, 2005, 186 p. dactyl. (www.atlas-patrimoine93.fr/ pg-html/etudes.php).

2. Lettre à en-tête des établissements Kalker (58 rue de Paris, 2 à 16 rue des Bruyères), 10 juillet 1946. Ville des Lilas, Direction du développement durable (DDD), PC 1943-1948.

3. Collection Jean Huret, Les Lilas.

4. Voir dans la base Mérimée (architecture) : notice IA93000624.

5. Ville des Lilas, DD , PC 1968 (1).

6. Voir dans la base Mérimée (architecture) : notice IA93000623.

7. Ville des Lilas, DDD, PC 1963 (1).

8. BOURNON, Fernand. Etat des communes, Les Lilas. Montévrain : Département de la Seine, 1903, p. 90.

9. L'entreprise Patrelle est mentionnée dans l'Annuaire du commerce en 1870.

10. Patrelle, 30, rue du Garde-Chasse et 17, avenue Waldeck-Rousseau, plan daté du 30 mai 1926, joint à une demande de permis de construire en date du 22 juillet 1944, suite au bombardement survenu dans la nuit du 18 au 19 avril 1944. Ville des Lilas, (DDD), PC 1943-1948.

11. Ville des Lilas, DDD, PD 1982/25 et 25 bis, PC 1983/39.

12. Voir dans la base Mérimée (architecture) : notices IA93000606 et IA93000622.

13. HURET, Jean. Quand les Lilas... Histoire de la colline des Lilas et de ses occupants au cours des âges. Les Lilas : Ville des Lilas, 1993, p. 146-157.

14. DECOUX Jérôme. Montreuil, patrimoine industriel. Paris : APPIF, coll. «Itinéraires du patrimoine $» \mathrm{n}^{\circ} 277,2003$.

15. Annuaire du Commerce et Bottin du commerce en premier lieu, offrant une série continue (1870-1980, dépouillement tous les 5 ans) puis, ponctuellement, l'Annuaire Sageret (1875, 1879, 1882, 1936, 1942) et l'Indicateur Bijou (1941, 1960).

16. Conservés aux Archives départementales de la Seine-Saint-Denis (AD Seine-Saint-Denis).

17. Les entreprises ayant changé de nom, mais conservant la même activité, n'entrent pas dans ce décompte.

18. HURET, Jean. Quand les Lilas... Histoire de la colline des Lilas et de ses occupants au cours des âges. Les Lilas : Ville des Lilas, 1993, p. 146-157.

19. HURET, Jean. Quand les Lilas... Histoire de la colline des Lilas et de ses occupants au cours des âges. Les Lilas : Ville des Lilas, 1993, p. 146.

20. Voir dans la base Mérimée (architecture) : notice IA93000619.

21. AD Seine-Saint-Denis, matrices cadastrales anciennes, vol. 319, case 645.

22. Voir dans la base Mérimée (architecture) : notice IA93000596.

23. Voir dans la base Mérimée (architecture) : notice IA93000592.

24. Voir dans la base Mérimée (architecture) : notice IA93000593. 
25. Inventaire général des monuments et richesses artistiques de la France, Montreuil, dossier collectif « usines » par Jérôme Decoux, sous la dir. de Dominique Hervier et Catherine Chaplain, 2000-2002.

26. A consulter sur www.culture.gouv.fr/documentation/merimee/accueil.htm et http:// www.atlas-patrimoine93.fr/.

27. Voir dans la base Mérimée (architecture) : notice IA93000606.

28. Voir dans la base Mérimée (architecture) : notice IA93000622.

29. Voir dans la base Mérimée (architecture) : notice IA93000597.

30. Voir dans la base Mérimée (architecture) : notice IA93000617.

31. Voir dans la base Mérimée (architecture) : notice IA93000609.

32. Voir dans la base Mérimée (architecture) : notice IA93000619.

33. Témoignage de Mme Hélène Goldschmidt, le 3 mars 2005.

34. Voir dans la base Mérimée (architecture) : notice IA93000603.

35. Voir dans la base Mérimée (architecture) : notice IA93000623.

36. Voir dans la base Mérimée (architecture) : notice IA93000602.

37. Témoignage de M. Jean-Pierre Piattino, le $1^{\mathrm{er}}$ février 2005.

38. DDD, PC 1946-1947 et 1949(2).

39. Voir dans la base Mérimée (architecture) : notice IA93000616.

40. Informations issues du dépouillement de l'Annuaire du commerce et du Bottin du commerce.

41. Voir dans la base Mérimée (architecture) : notice IA93000608.

42. AD Seine-Saint-Denis, $1322 \mathrm{~W} 160$ et $1328 \mathrm{~W} 77$.

43. AD Seine-Saint-Denis, $1322 \mathrm{~W} 160$ et $1328 \mathrm{~W} 77$.

44. Voir dans la base Mérimée (architecture) : notice IA93000600.

45. Voir dans la base Mérimée (architecture) : notice IA93000594.

46. Ville des Lilas, DDD, PC 1949(1) et 1951.

47. Ville des Lilas, DDD, PC 1951.

48. AD Seine-Saint-Denis, « Matrices cadastrales anciennes », vol. 318, case 1012.

49. Mandat de la société Ohresser, avec en-tête illustré, daté du 28 février 1934. Archives municipales des Lilas, non coté.

50. Voir dans la base Mérimée (architecture) : notice IA93000596.

51. Bottin du commerce, 1925.

52. Voir dans la base Mérimée (architecture) : notice IA93000598.

53. Voir dans la base Mérimée (architecture) : notice IA93000620.

54. AD Seine-Saint-Denis, 1322 W82.

55. Voir dans la base Mérimée (architecture) : notice IA93000604.

56. Voir dans la base Mérimée (architecture) : notice IA93000606.

57. Voir dans la base Mérimée (architecture) : notice IA93000597.

58. Ville des Lilas, DDD, PC 11/82.

59. Voir dans la base Mérimée (architecture) : notice IA93000592.

60. AD Seine-Saint-Denis, 219 W 37.

61. "L'idée consistait à rendre solidaires l'arbalétrier et le poteau de support en les raccordant par un arc de cercle, de façon à former une seule poutre continue triangulée. La poussée de la ferme se trouvait ainsi réduite à une force qui s'exerçait obliquement à la base du poteau, permettant ainsi la suppression totale des tirants, pourvu que le poteau ait été convenablement encastré dans le massif de fondation en maçonnerie.» (LEMOINE, Bertrand. L'Architecture du fer. France, XIXe siècle. Paris : Champ Vallon, 1986, p. 76).

62. Ville des Lilas, PC 1950(1).

63. Voir dans la base Mérimée (architecture) : notice IA93000593.

64. Suite au repérage du patrimoine industriel conduit par le CAUE 93 (Cécile Katz) au cours des années 1980 et 1990, le Bureau du patrimoine a réalisé l'inventaire du patrimoine industriel des 
communes de Pantin et de Bagnolet (Antoine Furio), et le service de l'Inventaire d'Ile-de-France ceux des communes de Montreuil (Jérôme Decoux) et du Pré-Saint-Gervais (Nicolas Pierrot).

65. LE ROUX, Thomas (dir.). La Maison des métallos et le Bas Belleville. Histoire et patrimoine industriel à Paris. Paris : Créaphis, 2003.

\section{RÉSUMÉS}

Les usines et les ateliers artisanaux repérés sur le territoire de la commune des Lilas ont été construits au cours du XXe siècle. A la différence de La Plaine-Saint-Denis, de ses industries lourdes, notamment chimiques et métallurgiques, à fortes emprises et génératrices d'emplois exigeant peu de qualification, Les Lilas accueillent à partir de la seconde moitié du XIXe siècle, à l'image des autres communes bordant Paris à l'est, des activités diversifiées recourant souvent à une main-d'œuvre qualifiée. Les entreprises, installées en grand nombre, sont ici disséminées en ateliers discrets: l'activité de production s'affirme peu, aux Lilas, par le décor. L'étude morphologique et architecturale permet de suivre l'évolution des ateliers et des usines, au fond des cours, le long des sentes, parfois sur la rue, et de comprendre les modalités de leur inscription dans le tissu urbain.

The factories and artisans' workshops surveyed in the suburban town of Les Lilas were built essentially during the XXth century. Unlike the town of La Plaine-Saint-Denis, whose heavy industry - namely chemical and metallurgical - generated a large unskilled workforce, Les Lilas attracted, from the second half of the XIXth century onward, numerous and varied industries that hired skilled workers. These companies were disseminated throughout the town in discreet workshops: ornate factories are not a "Lilasian" tradition. A morphological and architectural study allows us to follow the evolution of the workshops and factories found in back courtyards, along alleys or sometimes more visibly located, and to understand the different ways they wove themselves into the urban fabric of Les Lilas.

\section{INDEX}

Mots-clés : architecture industrielle, artisanat, atelier, banlieue parisienne, construction mécanique, désindustrialisation, entrepôts, habillement, industrialisation, Les Lilas (Seine-SaintDenis), métallurgie, paysage industriel, petite industrie, petite métallurgie, PLU (Plan local d'urbanisme), renouvellement urbain, textile, travail du bois, travail du cuir

Keywords : clothing trade, factory, industrial architecture, industrial landscape, leather working, mechanical engineering, metallurgy, Paris suburbs, small-scale industry, textile industry, urban renewal, warehouses, woodworking, workshop

\section{AUTEUR}

\section{NICOLAS PIERROT}

Ingénieur d'études. Service chargé de l'inventaire général du patrimoine culturel, Région Ile-deFrance.nicolas.pierrot@iledefrance.fr 